\title{
Coupled fixed point theorems for asymptotically nonexpansive mappings
}

\author{
Hallowed Olaoluwa ${ }^{1 *}$, Johnson O Olaleru ${ }^{1}$ and Shih-Sen Chang ${ }^{2}$
}

\section{*Correspondence:}

olu20_05@hotmail.com

'Department of Mathematics,

University of Lagos, Akoka, Lagos,

Nigeria

Full list of author information is

available at the end of the article

\begin{abstract}
We introduce the theory of asymptotical nonexpansiveness of mappings defined in the algebraic product $E \times E$ and with values in the space $E$. We then prove the existence of coupled fixed points of such mappings when $E$ is a uniformly convex Banach space. This paper is an extension of some recent results in the literature. MSC: $47 \mathrm{H} 10 ; 47 \mathrm{H} 09$

Keywords: coupled fixed point; nonexpansive map
\end{abstract}

\section{Introduction and preliminaries}

In the past years, many researchers have proved various results on the theory of nonexpansive mappings (or contractions). The mean ergodic theorem for contractions in uniformly convex Banach spaces was proved in [1], while the authors in [2] introduced the convex approximation property of a space, proved that contractions satisfy an inequality analogue to the Zarantonello inequality (see [3]) and then studied the asymptotic behavior of contractions.

Given a nonempty subset $D$ of a real linear normed space $E$, a self-mapping $T: D \rightarrow D$ is said to be nonexpansive if the following inequality holds for all $x, y \in D$ :

$$
\|T x-T y\| \leq\|x-y\| .
$$

Many more general classes of mappings have been considered, including the class of asymptotically nonexpansive mappings introduced by Goebel and Kirk [4], defined by the relation

$$
\left\|T^{n} x-T^{n} y\right\| \leq k_{n}\|x-y\| \quad \forall n \geq 1, \forall x, y \in D,
$$

where the sequence $\left\{k_{n}\right\} \subset[1,+\infty)$ converges to 1 as $n \rightarrow+\infty$. They proved that a self asymptotically nonexpansive map of a nonempty closed convex bounded subset of a real uniformly convex Banach space has a fixed point. Then Chang et al. [5] established some convergence theorems for this class of mappings without the assumption of boundedness of the subset $D$.

Recently, the concept of coupled fixed points was introduced and developed by some authors (see [6-8]). A coupled fixed point of a map $F: E \times E \rightarrow E$ is defined as an element $(x, y) \in E \times E$ such that $x=F(x, y)$ and $y=F(y, x)$. One could be interested in extending 
nonexpansiveness to maps defined on a product space (the algebraic product) and study the existence of their coupled fixed points. This is the main purpose of this paper.

We now introduce the definitions of nonexpansive maps, asymptotically nonexpansive maps, Lipschitzian and uniformly Lipschitzian maps defined in product spaces.

Definition 1.1 Let $D$ be a nonempty subset of a real normed linear space $E$. A mapping $F: D \times D \rightarrow D$ is said to be nonexpansive if

$$
\|F(x, y)-F(u, v)\| \leq \frac{1}{2}[\|x-u\|+\|y-v\|] \quad \forall x, y, u, v \in X .
$$

Definition 1.2 $F$ is said to be asymptotically nonexpansive if there exists a sequence $\left\{k_{n}\right\} \subset[1,+\infty)$ with $\lim _{n \rightarrow+\infty} k_{n}=1$ such that

$$
\left\|F^{n}(x, y)-F^{n}(u, v)\right\| \leq \frac{k_{n}}{2}[\|x-u\|+\|y-v\|] \quad \forall n \geq 1, \forall x, y, u, v \in X,
$$

where the sequence $\left\{F^{n}\right\}$ is defined (see [7]) as follows:

$$
\left\{\begin{array}{l}
F^{0}(x, y)=x, \\
F^{n+1}(x, y)=F\left(F^{n}(x, y), F^{n}(y, x)\right), \quad n \geq 0 .
\end{array}\right.
$$

Definition 1.3 $F$ is said to be uniformly L-Lipschitzian (where $L$ is a positive constant) if

$$
\left\|F^{n}(x, y)-F^{n}(u, v)\right\| \leq \frac{L}{2}[\|x-u\|+\|y-v\|] \quad \forall n \geq 1, \forall x, y, u, v \in X .
$$

When the equality is verified for $n=1$, i.e., when

$$
\|F(x, y)-F(u, v)\| \leq \frac{L}{2}[\|x-u\|+\|y-v\|] \quad \forall x, y, u, v \in X,
$$

$F$ is said to be Lipschitz with the constant $L$ (or L-Lipschitzian).

\section{Remark 1.4}

1. It is easy to see that if $F: D \times D \rightarrow D$ is a nonexpansive mapping, then $F$ is an asymptotically nonexpansive mapping with a constant sequence $\{1\}$.

2. If $T: D \times D \rightarrow D$ is an asymptotically nonexpansive mapping with a sequence $\left\{k_{n}\right\} \in[1,+\infty)$ such that $k_{n} \rightarrow 1$, then it must be uniformly $L$-Lipschitzian with $L=\sup _{n \geq 1}\left\{k_{n}\right\}$.

3. The sequence $\left\{F^{n}(x, y)\right\}$ can be written as the sequence $\left\{x_{n}\right\}$ defined (see [6]) as follows:

$$
\left\{\begin{array}{l}
x_{0}=x, \quad y_{0}=y, \\
x_{n+1}=F\left(x_{n}, y_{n}\right), \quad n \geq 0, \\
y_{n+1}=F\left(y_{n}, x_{n}\right), \quad n \geq 0 .
\end{array}\right.
$$

In [5], Chang et al. defined demi-closed maps at the origin as follows.

Definition 1.5 [5] Let $E$ be a real Banach space and $D$ be a closed subset of $E$. A mapping $T: D \rightarrow D$ is said to be demi-closed at the origin if, for any sequence $\left\{x_{n}\right\}$ in $D$, the conditions $x_{n} \rightarrow q$ weakly and $T x_{n} \rightarrow 0$ strongly imply $T q=0$. 
We extend this definition to maps defined in $D \times D$ as follows.

Definition 1.6 Let $E$ be a real Banach space and $D$ be a closed subset of $E$. A mapping $F: D \times D \rightarrow D$ is said to be demi-closed at the origin if, for any sequence $\left\{\left(x_{n}, y_{n}\right)\right\}$ in $D \times D$, the conditions $x_{n} \rightarrow q_{1}, y_{n} \rightarrow q_{2}$ weakly and $F\left(x_{n}, y_{n}\right) \rightarrow 0, F\left(y_{n}, x_{n}\right) \rightarrow 0$ strongly imply $F\left(q_{1}, q_{2}\right)=F\left(q_{2}, q_{1}\right)=0$.

Lemma 1.7 Let E be a uniformly convex Banach space, $C$ be a nonempty bounded closed convex subset of $E$. Then there exists a strictly increasing continuous convex function $f:[0,+\infty) \rightarrow[0,+\infty)$ with $f(0)=0$ such that for any Lipschitzian mapping $T: C \times C \rightarrow E$ with the Lipschitz constant $L \geq 1$, any finite many elements $\left\{\left(x_{i}, y_{i}\right)\right\}_{i=1}^{n}$ in $C \times C$ and any finite many nonnegative numbers $\left\{t_{i}\right\}_{i=1}^{n}$ with $\sum_{i=1}^{n} t_{i}=1$, the following inequality holds:

$$
\begin{aligned}
& \left\|T\left(\sum_{i=1}^{n} t_{i}\left(x_{i}, y_{i}\right)\right)-\sum_{i=1}^{n} t_{i} T\left(x_{i}, y_{i}\right)\right\| \\
& \quad \leq \frac{L}{2} f^{-1}\left\{\max _{1 \leq i, j \leq n}\left(\left\|x_{i}-x_{j}\right\|+\left\|y_{i}-y_{j}\right\|-\left(\frac{L}{2}\right)^{-1}\left\|T\left(x_{i}, y_{i}\right)-T\left(x_{j}, y_{j}\right)\right\|\right)\right\} .
\end{aligned}
$$

Proof Let us prove by induction. For $n=1,(1.7)$ is trivial.

Let us prove for $n=2$ : Let $\delta$ be the modulus of uniform convexity of $E$ and define $d$ : $\mathbb{R}^{+} \rightarrow \mathbb{R}^{+}$by

$$
d(t)=\left\{\begin{array}{l}
\frac{1}{2} \int_{0}^{t} \delta(s) d s, \quad 0 \leq t \leq 2 \\
d(2)+\frac{1}{2} \delta(2)(t-2), \quad t>2 .
\end{array}\right.
$$

It is well known $(e . g$., see $[1,9])$ that $d$ is strictly increasing, continuous, convex, satisfying $d(0)=0$ and

$$
2 t_{1} t_{2} d(\|u-v\|) \leq 1-\left\|t_{1} u+t_{2} v\right\|
$$

for all $t_{1}, t_{2} \geq 0$ such that $t_{1}+t_{2}=1$ and $u, v \in E$ such that $\|u\| \leq 1$ and $\|v\| \leq 1$.

$$
\begin{aligned}
\| T & \left(x_{1}, y_{1}\right)-T\left(\sum_{i=1}^{2} t_{i}\left(x_{i}, y_{i}\right)\right) \| \\
& =\left\|T\left(x_{1}, y_{1}\right)-T\left(t_{1} x_{1}+t_{2} x_{2}, t_{1} y_{1}+t_{2} y_{2}\right)\right\| \\
& \leq \frac{L}{2}\left\{\left\|x_{1}-t_{1} x_{1}-t_{2} x_{2}\right\|+\left\|y_{1}-t_{1} y_{1}-t_{2} y_{2}\right\|\right\} \\
& =\frac{L}{2}\left\{\left\|\left(1-t_{1}\right) x_{1}-t_{2} x_{2}\right\|+\left\|\left(1-t_{1}\right) y_{1}-t_{2} y_{2}\right\|\right\} \\
& =\frac{L}{2}\left\{\left\|t_{2} x_{1}-t_{2} x_{2}\right\|+\left\|t_{2} y_{1}-t_{2} y_{2}\right\|\right\} \\
& =\frac{L}{2} t_{2}\left[\left\|x_{1}-x_{2}\right\|+\left\|y_{1}-y_{2}\right\|\right] .
\end{aligned}
$$


Similarly,

$$
\begin{aligned}
\left\|T\left(x_{2}, y_{2}\right)-T\left(\sum_{i=1}^{2} t_{i}\left(x_{i}, y_{i}\right)\right)\right\| & =\left\|T\left(x_{2}, y_{2}\right)-T\left(t_{1} x_{1}+t_{2} x_{2}, t_{1} y_{1}+t_{2} y_{2}\right)\right\| \\
& \leq \frac{L}{2}\left\{\left\|x_{2}-t_{1} x_{1}-t_{2} x_{2}\right\|+\left\|y_{2}-t_{1} y_{1}-t_{2} y_{2}\right\|\right\} \\
& =\frac{L}{2}\left\{\left\|\left(1-t_{2}\right) x_{2}-t_{1} x_{1}\right\|+\left\|\left(1-t_{2}\right) y_{2}-t_{1} y_{1}\right\|\right\} \\
& =\frac{L}{2}\left\{\left\|t_{1} x_{2}-t_{1} x_{1}\right\|+\left\|t_{1} y_{2}-t_{1} y_{1}\right\|\right\} \\
& =\frac{L}{2} t_{1}\left[\left\|x_{1}-x_{2}\right\|+\left\|y_{1}-y_{2}\right\|\right] .
\end{aligned}
$$

Hence, if $u=\frac{T\left(x_{2}, y_{2}\right)-T\left(\sum_{i=1}^{2} t_{i}\left(x_{i}, y_{i}\right)\right)}{\frac{L}{2} t_{1}\left[\left\|x_{1}-x_{2}\right\|+\left\|y_{1}-y_{2}\right\|\right]}$ and $v=\frac{T\left(\sum_{i=1}^{2} t_{i}\left(x_{i}, y_{i}\right)\right)-T\left(x_{1}, y_{1}\right)}{\left.\frac{L}{2} t_{2}\|\| x_{1}-x_{2}\|+\| y_{1}-y_{2} \|\right]}$, then from (1.9) and (1.10),

$$
\left\{\begin{array}{l}
\|u\| \leq 1, \quad\|v\| \leq 1, \\
t_{1} u+t_{2} v=\frac{T\left(x_{2}, y_{2}\right)-T\left(x_{1}, y_{1}\right)}{\frac{L}{2}\left[\left\|x_{1}-x_{2}\right\|+\left\|y_{1}-y_{2}\right\|\right]}, \\
u-v=\frac{t_{1} T\left(x_{1}, y_{1}\right)+t_{2} T\left(x_{2}, y_{2}\right)-T\left(\sum_{i=1}^{2} t_{i}\left(x_{i}, y_{i}\right)\right)}{\frac{L}{2} t_{1} t_{2}\left[\left\|x_{1}-x_{2}\right\|+\left\|y_{1}-y_{2}\right\|\right]} .
\end{array}\right.
$$

Putting (1.11) in (1.8), we have

$$
2 t_{1} t_{2} d\left(\frac{\left\|t_{1} T\left(x_{1}, y_{1}\right)+t_{2} T\left(x_{2}, y_{2}\right)-T\left(\sum_{i=1}^{2} t_{i}\left(x_{i}, y_{i}\right)\right)\right\|}{\frac{L}{2} t_{1} t_{2}\left[\left\|x_{1}-x_{2}\right\|+\left\|y_{1}-y_{2}\right\|\right]}\right) \leq 1-\frac{\left\|T\left(x_{2}, y_{2}\right)-T\left(x_{1}, y_{1}\right)\right\|}{\frac{L}{2}\left[\left\|x_{1}-x_{2}\right\|+\left\|y_{1}-y_{2}\right\|\right]},
$$

i.e.,

$$
\begin{aligned}
& t_{1} t_{2} L\left[\left\|x_{1}-x_{2}\right\|+\left\|y_{1}-y_{2}\right\|\right] d\left(\frac{\left\|t_{1} T\left(x_{1}, y_{1}\right)+t_{2} T\left(x_{2}, y_{2}\right)-T\left(\sum_{i=1}^{2} t_{i}\left(x_{i}, y_{i}\right)\right)\right\|}{\frac{L}{2} t_{1} t_{2}\left[\left\|x_{1}-x_{2}\right\|+\left\|y_{1}-y_{2}\right\|\right]}\right) \\
& \quad \leq \frac{L}{2}\left[\left\|x_{1}-x_{2}\right\|+\left\|y_{1}-y_{2}\right\|\right]-\left\|T\left(x_{2}, y_{2}\right)-T\left(x_{1}, y_{1}\right)\right\| .
\end{aligned}
$$

Since $t \rightarrow \frac{d(t)}{t}$ is strictly increasing, $t_{1} t_{2} \leq \frac{1}{4}$ and $\left\|x_{1}-x_{2}\right\|+\left\|y_{1}-y_{2}\right\| \leq 2 D$, where $D:=$ $\operatorname{diam} C$, we have

$$
\begin{aligned}
& \frac{L}{2} D d\left(\frac{\left\|t_{1} T\left(x_{1}, y_{1}\right)+t_{2} T\left(x_{2}, y_{2}\right)-T\left(\sum_{i=1}^{2} t_{i}\left(x_{i}, y_{i}\right)\right)\right\|}{\frac{L}{4} D}\right) \\
& \quad \leq t_{1} t_{2} L\left[\left\|x_{1}-x_{2}\right\|+\left\|y_{1}-y_{2}\right\|\right] d\left(\frac{\left\|t_{1} T\left(x_{1}, y_{1}\right)+t_{2} T\left(x_{2}, y_{2}\right)-T\left(\sum_{i=1}^{2} t_{i}\left(x_{i}, y_{i}\right)\right)\right\|}{\frac{L}{2} t_{1} t_{2}\left[\left\|x_{1}-x_{2}\right\|+\left\|y_{1}-y_{2}\right\|\right]}\right) \\
& \quad \leq \frac{L}{2}\left[\left\|x_{1}-x_{2}\right\|+\left\|y_{1}-y_{2}\right\|\right]-\left\|T\left(x_{2}, y_{2}\right)-T\left(x_{1}, y_{1}\right)\right\| .
\end{aligned}
$$

Let $f_{2}: \mathbb{R}^{+} \rightarrow \mathbb{R}^{+}$be defined as $f_{2}(t)=D d\left(\frac{2 t}{D}\right)$; then

$$
\begin{aligned}
& \frac{L}{2} f_{2}\left(\frac{2}{L}\left\|t_{1} T\left(x_{1}, y_{1}\right)+t_{2} T\left(x_{2}, y_{2}\right)-T\left(\sum_{i=1}^{2} t_{i}\left(x_{i}, y_{i}\right)\right)\right\|\right) \\
& \quad \leq \frac{L}{2}\left[\left\|x_{1}-x_{2}\right\|+\left\|y_{1}-y_{2}\right\|\right]-\left\|T\left(x_{2}, y_{2}\right)-T\left(x_{1}, y_{1}\right)\right\| .
\end{aligned}
$$


Hence,

$$
\begin{aligned}
& \left\|t_{1} T\left(x_{1}, y_{1}\right)+t_{2} T\left(x_{2}, y_{2}\right)-T\left(\sum_{i=1}^{2} t_{i}\left(x_{i}, y_{i}\right)\right)\right\| \\
& \quad \leq \frac{L}{2} f_{2}^{-1}\left(\left\|x_{1}-x_{2}\right\|+\left\|y_{1}-y_{2}\right\|-\frac{2}{L}\left\|T\left(x_{2}, y_{2}\right)-T\left(x_{1}, y_{1}\right)\right\|\right) .
\end{aligned}
$$

Thus (1.7) is true for $n=2$.

Now suppose that

$$
\begin{aligned}
& \left\|T\left(\sum_{i=1}^{n} t_{i}\left(x_{i}, y_{i}\right)\right)-\sum_{i=1}^{n} t_{i} T\left(x_{i}, y_{i}\right)\right\| \\
& \quad \leq \frac{L}{2} f_{n}^{-1}\left\{\max _{1 \leq i, j \leq n}\left(\left\|x_{i}-x_{j}\right\|+\left\|y_{i}-y_{j}\right\|-\left(\frac{L}{2}\right)^{-1}\left\|T\left(x_{i}, y_{i}\right)-T\left(x_{j}, y_{j}\right)\right\|\right)\right\} .
\end{aligned}
$$

Define a strictly increasing continuous convex function $f_{n+1}$ satisfying $f_{n+1}(0)=0$ and

$$
f_{n+1}^{-1}(t) \geq f_{2}^{-1}(t)+f_{n}^{-1}\left(t+2 f_{2}^{-1}(t)\right)
$$

For example, if $*$ defines the inf-convolution, $I$ the identity function on $\mathbb{R}$ and the functions in the functions $f_{n}$ are extended to be $+\infty$ on $(-\infty, 0)$, then one could take (see [2])

$$
f_{n+1}=\frac{1}{2}\left(I * f_{2}\right) o\left(\frac{1}{2}\left(f_{2} * f_{n}\right)\right) \text {. }
$$

Define also $\Delta^{n-1}=\left\{t=\left(t_{1}, \ldots, t_{n}\right): t_{i} \geq 0, \sum_{i=1}^{n} t_{i}=1\right\}$.

Fix $t \in \Delta^{n}$ and let $\left(x_{1}, y_{1}\right), \ldots,\left(x_{n+1}, y_{n+1}\right) \in C \times C$. The case $t_{n+1}=1$ is trivial and therefore omitted. For the rest of the proof, we let the subscript $i$ range through $1 \leq i \leq n+1$, while $j$ ranges through $1 \leq j \leq n$. Put

$$
\begin{aligned}
& \left(\begin{array}{c}
u_{j} \\
v_{j}
\end{array}\right)=\left(1-t_{n+1}\right)\left(\begin{array}{l}
x_{j} \\
y_{j}
\end{array}\right)+t_{n+1}\left(\begin{array}{c}
x_{n+1} \\
y_{n+1}
\end{array}\right), \\
& \left(\begin{array}{l}
x_{i}^{\prime} \\
y_{i}^{\prime}
\end{array}\right)=\left(\begin{array}{l}
T\left(x_{i}, y_{i}\right) \\
T\left(y_{i}, x_{i}\right)
\end{array}\right), \\
& \left(\begin{array}{c}
u_{j}^{\prime} \\
v_{j}^{\prime}
\end{array}\right)=\left(1-t_{n+1}\right)\left(\begin{array}{c}
x_{j}^{\prime} \\
y_{j}^{\prime}
\end{array}\right)+t_{n+1}\left(\begin{array}{c}
x_{n+1}^{\prime} \\
y_{n+1}^{\prime}
\end{array}\right), \\
& \mu_{j}=\frac{t_{j}}{1-t_{n+1}} .
\end{aligned}
$$

We have that $\mu \in \Delta^{n-1}$ and after computation

$$
\sum_{i=1}^{n+1} t_{i}\left(\begin{array}{l}
x_{i} \\
y_{i}
\end{array}\right)=\sum_{j=1}^{n} \mu_{j}\left(\begin{array}{l}
u_{j} \\
v_{j}
\end{array}\right), \quad \sum_{i=1}^{n+1} t_{i}\left(\begin{array}{l}
x_{i}^{\prime} \\
y_{i}^{\prime}
\end{array}\right)=\sum_{j=1}^{n} \mu_{j}\left(\begin{array}{l}
u_{j}^{\prime} \\
v_{j}^{\prime}
\end{array}\right),
$$


The induction hypothesis (1.13) can also be written

$$
\begin{aligned}
f_{n} & \left(\frac{2}{L}\left\|T\left(\sum_{j=1}^{n} \mu_{j}\left(u_{j}, v_{j}\right)\right)-\sum_{j=1}^{n} \mu_{j} T\left(u_{j}, v_{j}\right)\right\|\right) \\
\leq & \max _{1 \leq j, k \leq n}\left(\left\|u_{j}-u_{k}\right\|+\left\|v_{j}-v_{k}\right\|-\frac{2}{L}\left\|T\left(u_{j}, v_{j}\right)-T\left(u_{k}, v_{k}\right)\right\|\right) \\
= & \max _{1 \leq j, k \leq n}\left(\left\|u_{j}-u_{k}\right\|+\left\|v_{j}-v_{k}\right\|-\frac{2}{L}\left\|T\left(v_{j}, u_{j}\right)-T\left(v_{k}, u_{k}\right)\right\|\right) \\
= & \max _{1 \leq j, k \leq n}\left(\left\|u_{j}-u_{k}\right\|+\left\|v_{j}-v_{k}\right\|-\frac{1}{L}\left\|T\left(u_{j}, v_{j}\right)-T\left(u_{k}, v_{k}\right)\right\|\right. \\
& \left.-\frac{1}{L}\left\|T\left(v_{j}, u_{j}\right)-T\left(v_{k}, u_{k}\right)\right\|\right) .
\end{aligned}
$$

We also have, by the triangle inequality,

$$
\begin{aligned}
& \left\|u_{j}-u_{k}\right\|-\frac{1}{L}\left\|T\left(u_{j}, v_{j}\right)-T\left(u_{k}, v_{k}\right)\right\| \\
& \leq\left\|u_{j}-u_{k}\right\|-\frac{1}{L}\left\|u_{j}^{\prime}-u_{k}^{\prime}\right\|+\frac{1}{L}\left\|u_{k}^{\prime}-T\left(u_{k}, v_{k}\right)\right\|+\frac{1}{L}\left\|u_{j}^{\prime}-T\left(u_{j}, v_{j}\right)\right\|, \\
& \left\|v_{j}-v_{k}\right\|-\frac{1}{L}\left\|T\left(v_{j}, u_{j}\right)-T\left(v_{k}, u_{k}\right)\right\| \\
& \quad \leq\left\|v_{j}-v_{k}\right\|-\frac{1}{L}\left\|v_{j}^{\prime}-v_{k}^{\prime}\right\|+\frac{1}{L}\left\|v_{k}^{\prime}-T\left(v_{k}, u_{k}\right)\right\|+\frac{1}{L}\left\|v_{j}^{\prime}-T\left(v_{j}, u_{j}\right)\right\| .
\end{aligned}
$$

From (1.12) we have

$$
\begin{aligned}
f_{2}\left(\frac{2}{L}\left\|T\left(u_{j}, v_{j}\right)-u_{j}^{\prime}\right\|\right)= & f_{2}\left(\frac{2}{L}\left\|T\left(u_{j}, v_{j}\right)-\left(1-t_{n+1}\right) x_{j}^{\prime}-t_{n+1} x_{n+1}^{\prime}\right\|\right) \\
= & f_{2}\left(\frac{2}{L}\left\|T\left(u_{j}, v_{j}\right)-\left(1-t_{n+1}\right) T\left(x_{j}, y_{j}\right)-t_{n+1} T\left(x_{n+1}, y_{n+1}\right)\right\|\right) \\
= & f_{2}\left(\frac{2}{L} \| T\left(\left(1-t_{n+1}\right) x_{j}+t_{n+1} x_{n+1},\left(1-t_{n+1}\right) y_{j}+t_{n+1} y_{n+1}\right)\right. \\
& \left.-\left(1-t_{n+1}\right) T\left(x_{j}, y_{j}\right)-t_{n+1} T\left(x_{n+1}, y_{n+1}\right) \|\right) \\
\leq & \left\|x_{j}-x_{n+1}\right\|+\left\|y_{j}-y_{n+1}\right\|-\frac{2}{L}\left\|T\left(x_{j}, y_{j}\right)-T\left(x_{n+1}, y_{n+1}\right)\right\| \\
= & \left\|x_{j}-x_{n+1}\right\|+\left\|y_{j}-y_{n+1}\right\|-\frac{2}{L}\left\|x_{j}^{\prime}-x_{n+1}^{\prime}\right\| .
\end{aligned}
$$


Similarly,

$$
f_{2}\left(\frac{2}{L}\left\|T\left(v_{j}, u_{j}\right)-v_{j}^{\prime}\right\|\right) \leq\left\|x_{j}-x_{n+1}\right\|+\left\|y_{j}-y_{n+1}\right\|-\frac{2}{L}\left\|y_{j}^{\prime}-y_{n+1}^{\prime}\right\| .
$$

We also have

$$
\begin{aligned}
\left\|u_{j}-u_{k}\right\|-\frac{1}{L}\left\|u_{j}^{\prime}-u_{k}^{\prime}\right\| & =\left\|\left(1-t_{n+1}\right)\left(x_{j}-x_{k}\right)\right\|-\frac{1}{L}\left\|\left(1-t_{n+1}\right)\left(x_{j}^{\prime}-x_{k}^{\prime}\right)\right\| \\
& =\left(1-t_{n+1}\right)\left[\left\|x_{j}-x_{k}\right\|-\frac{1}{L}\left\|x_{j}^{\prime}-x_{k}^{\prime}\right\|\right] \\
& \leq\left\|x_{j}-x_{k}\right\|-\frac{1}{L}\left\|x_{j}^{\prime}-x_{k}^{\prime}\right\| .
\end{aligned}
$$

Similarly,

$$
\left\|v_{j}-v_{k}\right\|-\frac{1}{L}\left\|v_{j}^{\prime}-v_{k}^{\prime}\right\| \leq\left\|y_{j}-y_{k}\right\|-\frac{1}{L}\left\|y_{j}^{\prime}-y_{k}^{\prime}\right\| .
$$

Put

$$
\begin{aligned}
t & :=\max \left\{\left\|x_{i}-x_{k}\right\|+\left\|y_{i}-y_{k}\right\|-\frac{2}{L}\left\|x_{i}^{\prime}-x_{k}^{\prime}\right\|: 1 \leq i, k \leq n+1\right\} \\
& =\max \left\{\left\|x_{i}-x_{k}\right\|+\left\|y_{i}-y_{k}\right\|-\frac{2}{L}\left\|y_{i}^{\prime}-y_{k}^{\prime}\right\|: 1 \leq i, k \leq n+1\right\} \\
& =\max \left\{\left\|x_{i}-x_{k}\right\|+\left\|y_{i}-y_{k}\right\|-\frac{1}{L}\left\|x_{i}^{\prime}-x_{k}^{\prime}\right\|-\frac{1}{L}\left\|y_{i}^{\prime}-y_{k}^{\prime}\right\|: 1 \leq i, k \leq n+1\right\} .
\end{aligned}
$$

Then, by (1.18) and (1.19),

$$
\frac{2}{L}\left\|T\left(u_{j}, v_{j}\right)-u_{j}^{\prime}\right\| \leq f_{2}^{-1}(t) \quad \text { and } \quad \frac{2}{L}\left\|T\left(v_{j}, u_{j}\right)-v_{j}^{\prime}\right\| \leq f_{2}^{-1}(t) .
$$

Using (1.20) and (1.22) in (1.16) and also (1.21) and (1.22) in (1.17), we have

$$
\left\|u_{j}-u_{k}\right\|-\frac{1}{L}\left\|T\left(u_{j}, v_{j}\right)-T\left(u_{k}, v_{k}\right)\right\| \leq\left\|x_{j}-x_{k}\right\|-\frac{1}{L}\left\|x_{j}^{\prime}-x_{k}^{\prime}\right\|+f_{2}^{-1}(t)
$$

and

$$
\left\|v_{j}-v_{k}\right\|-\frac{1}{L}\left\|T\left(v_{j}, u_{j}\right)-T\left(v_{k}, u_{k}\right)\right\| \leq\left\|y_{j}-y_{k}\right\|-\frac{1}{L}\left\|y_{j}^{\prime}-y_{k}^{\prime}\right\|+f_{2}^{-1}(t) ;
$$

by summing,

$$
\begin{aligned}
& \left\|u_{j}-u_{k}\right\|+\left\|v_{j}-v_{k}\right\|-\frac{1}{L}\left\|T\left(u_{j}, v_{j}\right)-T\left(u_{k}, v_{k}\right)\right\|-\frac{1}{L}\left\|T\left(v_{j}, u_{j}\right)-T\left(v_{k}, u_{k}\right)\right\| \\
& \quad \leq\left\|x_{j}-x_{k}\right\|+\left\|y_{j}-y_{k}\right\|-\frac{1}{L}\left\|x_{j}^{\prime}-x_{k}^{\prime}\right\|-\frac{1}{L}\left\|y_{j}^{\prime}-y_{k}^{\prime}\right\|+2 f_{2}^{-1}(t) \\
& \quad \leq t+2 f_{2}^{-1}(t) .
\end{aligned}
$$


Using (1.23) in (1.15) yields

$$
\left\|T\left(\sum_{j=1}^{n} \mu_{j}\left(u_{j}, v_{j}\right)\right)-\sum_{j=1}^{n} \mu_{j} T\left(u_{j}, v_{j}\right)\right\| \leq \frac{L}{2} f_{n}^{-1}\left(t+2 f_{2}^{-1}(t)\right) .
$$

Finally, when (1.22) and (1.24) are used in (1.14), we obtain

$$
\begin{aligned}
\left\|T\left(\sum_{i=1}^{n+1} t_{i}\left(x_{i}, y_{i}\right)\right)-\sum_{i=1}^{n+1} t_{i} T\left(x_{i}, y_{i}\right)\right\| & \leq \frac{L}{2} f_{n}^{-1}\left(t+2 f_{2}^{-1}(t)\right)+\sum_{j=1}^{n} \mu_{j} \frac{L}{2} f_{2}^{-1}(t) \\
& \leq \frac{L}{2} f_{n}^{-1}\left(t+2 f_{2}^{-1}(t)\right)+\frac{L}{2} f_{2}^{-1}(t) \\
& =\frac{L}{2}\left[f_{n}^{-1}\left(t+2 f_{2}^{-1}(t)\right)+f_{2}^{-1}(t)\right] \\
& \leq \frac{L}{2} f_{n+1}^{-1}(t) \quad \text { by the definition of } f_{n+1} .
\end{aligned}
$$

Therefore

$$
\begin{aligned}
& \left\|T\left(\sum_{i=1}^{n+1} t_{i}\left(x_{i}, y_{i}\right)\right)-\sum_{i=1}^{n+1} t_{i} T\left(x_{i}, y_{i}\right)\right\| \\
& \quad \leq \frac{L}{2} f_{n+1}^{-1}\left(\max _{1 \leq i, k \leq n+1}\left\{\left\|x_{i}-x_{k}\right\|+\left\|y_{i}-y_{k}\right\|-\frac{2}{L}\left\|T\left(x_{i}, y_{i}\right)-T\left(x_{k}, y_{k}\right)\right\|\right\}\right) .
\end{aligned}
$$

To complete the proof, we show in the sequel that the dependence of $f_{n}$ on $n$ can actually be omitted.

Since $E$ is uniformly convex, $E$ is $B$-convex (see [2]) and since the product of $B$-convex spaces is also $B$-convex (see [10]), $E^{3}$ is $B$-convex, hence has the convex approximation property (C.A.P.) (see [2]), i.e., for each $\epsilon>0$, there exists a positive integer $p$ such that

$$
\operatorname{co} M \subset \operatorname{co}_{p} M+S\left(0, \frac{\epsilon}{3 L}\right) \times S\left(0, \frac{\epsilon}{3 L}\right) \times S\left(0, \frac{\epsilon}{3 L}\right)
$$

where $S(0, r)$ is the open sphere centered at the origin and with $r$ as radius, $\operatorname{co} M$ is the convex hall of $M$ and

$$
\operatorname{co}_{p} M=\left\{\sum_{i=1}^{p} t_{i} X_{i} ; t \in \Delta^{p-1} ; X_{i} \in M \text { for all } i \in\{1, \ldots, p\}, p \text { fixed }\right\}
$$

for every $M \subset C \times C \times C$.

Put $\gamma=f_{p}\left(\frac{2 \epsilon}{3 L}\right)$. Suppose $x_{1}, \ldots, x_{n}, y_{1}, \ldots, y_{n} \in C$ satisfy

$$
\left\|x_{i}-x_{j}\right\|+\left\|y_{i}-y_{j}\right\|-\frac{2}{L}\left\|T\left(x_{i}, y_{i}\right)-T\left(x_{j}, y_{j}\right)\right\| \leq \gamma \quad \text { for all } i, j .
$$

Consider $M=\left\{\left(x_{i}, y_{i}, T\left(x_{i}, y_{i}\right)\right) \in C^{3}: i=1,2, \ldots, n\right\}$. Thus, for each $t \in \Delta^{n-1}$, there exist 
$\mu \in \Delta^{p-1}$ and $i_{1}, \ldots, i_{p} \in\{1, \ldots, n\}$ such that

$$
\begin{aligned}
& \left\|\sum_{i=1}^{n} t_{i} x_{i}-\sum_{j=1}^{p} \mu_{j} x_{i_{j}}\right\|<\frac{\epsilon}{3 L}, \\
& \left\|\sum_{i=1}^{n} t_{i} y_{i}-\sum_{j=1}^{p} \mu_{j} y_{i_{j}}\right\|<\frac{\epsilon}{3 L}, \\
& \left\|\sum_{i=1}^{n} t_{i} T\left(x_{i}, y_{i}\right)-\sum_{j=1}^{p} \mu_{j} T\left(x_{i_{j}}, y_{i_{j}}\right)\right\|<\frac{\epsilon}{3 L} \leq \frac{\epsilon}{3} .
\end{aligned}
$$

Now

$$
\begin{aligned}
\left\|T\left(\sum t_{i}\left(x_{i}, y_{i}\right)\right)-\sum t_{i} T\left(x_{i}, y_{i}\right)\right\| \leq & \left\|T\left(\sum t_{i}\left(x_{i}, y_{i}\right)\right)-T\left(\sum \mu_{j}\left(x_{i_{j}}, y_{i_{j}}\right)\right)\right\| \\
& +\left\|T\left(\sum \mu_{j}\left(x_{i_{j}}, y_{i_{j}}\right)\right)-\sum \mu_{j} T\left(x_{i_{j}}, y_{i_{j}}\right)\right\| \\
& +\left\|\sum \mu_{j} T\left(x_{i j}, y_{i_{j}}\right)-\sum t_{i} T\left(x_{i}, y_{i}\right)\right\| .
\end{aligned}
$$

Since

$$
\begin{aligned}
& \left\|T\left(\sum t_{i}\left(x_{i}, y_{i}\right)\right)-T\left(\sum \mu_{j}\left(x_{i_{j}}, y_{i_{j}}\right)\right)\right\| \\
& \quad \leq \frac{L}{2}\left[\left\|\sum t_{i} x_{i}-\sum \mu_{j} x_{i_{j}}\right\|+\left\|\sum t_{i} y_{i}-\sum \mu_{j} y_{i_{j}}\right\|\right] \\
& \quad<\frac{L}{2}\left[\frac{\epsilon}{3 L}+\frac{\epsilon}{3 L}\right]=\frac{\epsilon}{3}
\end{aligned}
$$

and

$$
\left\|T\left(\sum \mu_{j}\left(x_{i_{j}}, y_{i_{j}}\right)\right)-\sum \mu_{j} T\left(x_{i_{j}}, y_{i_{j}}\right)\right\| \leq \frac{L}{2} f_{p}^{-1}(\gamma)=\frac{L}{2} f_{p}^{-1}\left(f_{p}\left(\frac{2 \epsilon}{3 L}\right)\right)=\frac{\epsilon}{3}
$$

we have that

$$
\left\|T\left(\sum t_{i}\left(x_{i}, y_{i}\right)\right)-\sum t_{i} T\left(x_{i}, y_{i}\right)\right\|<\frac{\epsilon}{3}+\frac{\epsilon}{3}+\frac{\epsilon}{3}=\epsilon
$$

whenever $\left\|x_{i}-x_{j}\right\|+\left\|y_{i}-y_{j}\right\|-\frac{2}{L}\left\|T\left(x_{i}, y_{i}\right)-T\left(x_{j}, y_{j}\right)\right\| \leq \gamma$. One can easily construct $f$ such that $\epsilon<\frac{L}{2} f^{-1}(\gamma)$, i.e., such that $f(t) \leq f_{p}\left(\frac{t}{3}\right)$, which guarantees (1.7) with $f$ independent of $n$.

\section{Existence of coupled fixed points}

Let $p_{1}: E \times E \rightarrow E$ denote the projection of the first coordinate, i.e., $p_{1}(x, y)=x$ for all $x, y \in X$. Note that if $F: D \times D \rightarrow D$ is a mapping, we have that $p_{1}-F$ is demi-closed at the origin if the existence of a sequence $\left\{\left(x_{n}, y_{n}\right)\right\}$ in $D \times D$ such that

$$
\left\{\begin{array}{l}
x_{n} \rightarrow q_{1} \quad \text { weakly, } \\
y_{n} \rightarrow q_{2} \text { weakly, } \\
x_{n}-F\left(x_{n}, y_{n}\right) \rightarrow 0 \text { strongly, } \\
y_{n}-F\left(y_{n}, x_{n}\right) \rightarrow 0 \text { strongly }
\end{array}\right.
$$


implies that

$$
\left(p_{1}-F\right)\left(q_{1}, q_{2}\right)=\left(p_{1}-F\right)\left(q_{2}, q_{1}\right)=0,
$$

i.e.,

$$
F\left(q_{1}, q_{2}\right)=q_{1} \quad \text { and } \quad q_{2}=F\left(q_{2}, q_{1}\right)
$$

i.e., the existence of a coupled fixed point $\left(q_{1}, q_{2}\right)$ of $F$.

Now we prove our main theorem.

Theorem 2.1 Let $D$ be a nonempty closed convex subset of a uniformly convex Banach space $E$ and $F: D \times D \rightarrow D$ be an asymptotically nonexpansive map with the sequence $\left\{k_{n}\right\}$ as defined in (1.2). Then $p_{1}-F$ satisfies the demi-closedness at the origin property. In other terms, if any sequence $\left\{\left(x_{n}, y_{n}\right)\right\}$ in $D \times D$ is such that $x_{n} \rightarrow q_{1}$ weakly, $y_{n} \rightarrow q_{2}$ weakly, $x_{n}-F\left(x_{n}, y_{n}\right) \rightarrow 0$ strongly and $y_{n}-F\left(y_{n}, x_{n}\right) \rightarrow 0$ strongly, then $F$ has a coupled fixed point $\left(q_{1}, q_{2}\right)$.

Proof Since $\left\{\left(x_{n}, y_{n}\right)\right\}$ converges weakly to $q=\left(q_{1}, q_{2}\right) \in D \times D,\left\{x_{n}\right\}$ and $\left\{y_{n}\right\}$ are bounded in $D$. Therefore, there exists $r>0$ such that $\left\{x_{n}\right\},\left\{y_{n}\right\} \subset C=: D \cap B[0, r]$, where $B[0, r]$ is the ball of $E$ of radius $r$ centered in 0 . Hence $C$ is a nonempty bounded closed convex subset in $D$.

Next, we prove that as $n \rightarrow+\infty, F^{n} q \rightarrow q_{1}$ and $F^{n} \bar{q} \rightarrow q_{2}$, where $q=\left(q_{1}, q_{2}\right)$ and $\bar{q}=$ $\left(q_{2}, q_{1}\right)$.

Since $\left\{x_{n}\right\}$ and $\left\{y_{n}\right\}$ converge weakly to $q_{1}$ and $q_{2}$ respectively, by Mazur's theorem (see, e.g., [4]), for all $n \geq 1$, there exist sequences $\left\{A_{n}\right\}$ and $\left\{B_{n}\right\}$ such that $A_{n}=\sum_{i=1}^{m(n)} t_{i}^{(n)} x_{i+n}$ and $B_{n}=\sum_{i=1}^{m(n)} t_{i}^{(n)} y_{i+n}$, where $t_{i}^{(n)} \geq 0, \sum_{i=1}^{m(n)} t_{i}^{(n)}=1$ and $\left\|A_{n}-q_{1}\right\|<\frac{1}{n},\left\|B_{n}-q_{2}\right\|<\frac{1}{n}$.

Since the sequences $\left\{x_{n}-F\left(x_{n}, y_{n}\right)\right\}$ and $\left\{y_{n}-F\left(y_{n}, x_{n}\right)\right\}$ converge strongly to 0 respectively, for any given $\epsilon>0$ and positive integer $j \geq 1$, there is an integer $N=N(\epsilon, j)$ such that $\frac{1}{N}<\epsilon$ and

$$
\left\|x_{n}-F\left(x_{n}, y_{n}\right)\right\|+\left\|y_{n}-F\left(y_{n}, x_{n}\right)\right\| \leq \frac{1}{1+\sum_{l=1}^{j-1} k_{l} / 2}<\epsilon, \quad n \geq N
$$

Since $F$ is asymptotically nonexpansive,

$$
\begin{aligned}
\left\|F^{j}\left(x_{n}, y_{n}\right)-F^{j+1}\left(x_{n}, y_{n}\right)\right\| & =\left\|F^{j}\left(x_{n}, y_{n}\right)-F^{j}\left(F\left(x_{n}, y_{n}\right), F\left(y_{n}, x_{n}\right)\right)\right\| \\
& \leq \frac{k_{j}}{2}\left[\left\|x_{n}-F\left(x_{n}, y_{n}\right)\right\|+\left\|y_{n}-F\left(y_{n}, x_{n}\right)\right\|\right]
\end{aligned}
$$

and

$$
\begin{aligned}
\left\|F^{j}\left(y_{n}, x_{n}\right)-F^{j+1}\left(y_{n}, x_{n}\right)\right\| & =\left\|F^{j}\left(y_{n}, x_{n}\right)-F^{j}\left(F\left(y_{x}, y_{n}\right), F\left(x_{n}, y_{n}\right)\right)\right\| \\
& \leq \frac{k_{j}}{2}\left[\left\|x_{n}-F\left(x_{n}, y_{n}\right)\right\|+\left\|y_{n}-F\left(y_{n}, x_{n}\right)\right\|\right] .
\end{aligned}
$$


Hence, for any $n \geq N$,

$$
\begin{aligned}
\left\|x_{n}-F^{j}\left(x_{n}, y_{n}\right)\right\| \leq & \left\|x_{n}-F\left(x_{n}, y_{n}\right)\right\|+\left\|\left(F-F^{2}\right)\left(x_{n}, y_{n}\right)\right\|+\cdots \\
& +\left\|\left(F^{j-1}-F^{j}\right)\left(x_{n}, y_{n}\right)\right\| \\
\leq & \left(1+\sum_{l=1}^{j-1} k_{l} / 2\right)\left[\left\|x_{n}-F\left(x_{n}, y_{n}\right)\right\|+\left\|y_{n}-F\left(y_{n}, x_{n}\right)\right\|\right] \\
& <\epsilon .
\end{aligned}
$$

Similarly,

$$
\left\|y_{n}-F^{j}\left(y_{n}, x_{n}\right)\right\|<\epsilon .
$$

Since $F: D \times D \rightarrow D$ is asymptotically nonexpansive, $F: C \times C \rightarrow D$ is also asymptotically nonexpansive, hence $F^{j}: C \times C \rightarrow D$ is a Lipschitzian mapping with the Lipschitz constant $k_{j} \geq 1$. We have the following inequality:

$$
\begin{aligned}
\left\|F^{j}\left(A_{n}, B_{n}\right)-A_{n}\right\|= & F^{j}\left(A_{n}, B_{n}\right)-\sum_{i=1}^{m(n)} t_{i}^{(n)} F^{j}\left(x_{i+n}, y_{i+n}\right) \\
& +\sum_{i=1}^{m(n)} t_{i}^{(n)} F^{j}\left(x_{i+n}, y_{i+n}\right)-\sum_{i=1}^{m(n)} t_{i}^{(n)} x_{i+n} \| \\
\leq & \left\|F^{j}\left(A_{n}, B_{n}\right)-\sum_{i=1}^{m(n)} t_{i}^{(n)} F^{j}\left(x_{i+n}, y_{i+n}\right)\right\| \\
& +\sum_{i=1}^{m(n)} t_{i}^{(n)}\left\|F^{j}\left(x_{i+n}, y_{i+n}\right)-x_{i+n}\right\| .
\end{aligned}
$$

By (2.1), we know that

$$
\sum_{i=1}^{m(n)} t_{i}^{(n)}\left\|F^{j}\left(x_{i+n}, y_{i+n}\right)-x_{i+n}\right\|<\epsilon \quad \forall n \geq N
$$

By Lemma 1.7, (2.1) and (2.2), we have

$$
\begin{aligned}
&\left\|F^{j}\left(A_{n}, B_{n}\right)-\sum_{i=1}^{m(n)} t_{i}^{(n)} F^{j}\left(x_{i+n}, y_{i+n}\right)\right\| \\
& \leq\left(k_{j} / 2\right) f^{-1}\left\{\operatorname { m a x } _ { 1 \leq i , k \leq m ( n ) } \left(\left\|x_{i+n}-x_{k+n}\right\|+\left\|y_{i+n}-y_{k+n}\right\|\right.\right. \\
&\left.\left.-\left(k_{j} / 2\right)^{-1}\left\|F^{j}\left(x_{i+n}, y_{i+n}\right)-F^{j}\left(x_{k+n}, y_{k+n}\right)\right\|\right)\right\} \\
& \leq\left(k_{j} / 2\right) f^{-1}\left\{\operatorname { m a x } _ { 1 \leq i , k \leq m ( n ) } \left[\left\|x_{i+n}-F^{j}\left(x_{i+n}, y_{i+n}\right)\right\|+\left\|y_{i+n}-F^{j}\left(y_{i+n}, x_{i+n}\right)\right\|\right.\right. \\
&+\left\|F^{j}\left(x_{i+n}, y_{i+n}\right)-F^{j}\left(x_{k+n}, y_{k+n}\right)\right\|+\left\|F^{j}\left(y_{i+n}, x_{i+n}\right)-F^{j}\left(y_{k+n}, x_{k+n}\right)\right\| \\
&\left.+\left\|F^{j}\left(x_{k+n}, y_{k+n}\right)-x_{k+n}\right\|+\left\|F^{j}\left(y_{k+n}, x_{k+n}\right)-y_{k+n}\right\|\right]
\end{aligned}
$$




$$
\begin{aligned}
& \left.-\left(k_{j} / 2\right)^{-1}\left\|F^{j}\left(x_{i+n}, y_{i+n}\right)-F^{j}\left(x_{k+n}, y_{k+n}\right)\right\|\right\} \\
\leq & \left(k_{j} / 2\right) f^{-1}\left\{\max _{1 \leq i, k \leq m(n)}\left[4 \epsilon+\left(1-k_{j}^{-1}\right) k_{j}\left(\left\|x_{i+n}-x_{k+n}\right\|+\left\|y_{i+n}-y_{k+n}\right\|\right)\right]\right\} \\
\leq & \left(k_{j} / 2\right) f^{-1}\left(4 \epsilon+2 r\left(1-k_{j}^{-1}\right) k_{j}\right), \quad n \geq N .
\end{aligned}
$$

Inequalities (2.4) and (2.5) into (2.3) yield

$$
\left\|F^{j}\left(A_{n}, B_{n}\right)-A_{n}\right\| \leq\left(k_{j} / 2\right) f^{-1}\left[4 \epsilon+2 r k_{j}\left(2-k_{j}^{-1}\right)\right]+\epsilon .
$$

Taking the limit superior as $n \rightarrow+\infty$ in the above inequality and noting that $\epsilon>0$ is arbitrary, we have

$$
\limsup _{n \rightarrow+\infty}\left\|F^{j}\left(A_{n}, B_{n}\right)-A_{n}\right\| \leq\left(k_{j} / 2\right) f^{-1}\left[2 r k_{j}\left(2-k_{j}^{-1}\right)\right]
$$

Also, by the definition of the sequences $\left\{A_{n}\right\}$ and $\left\{B_{n}\right\}$, we have that for all $j \geq 1$,

$$
\begin{aligned}
\left\|F^{j}(q)-q_{1}\right\| & \leq\left\|F^{j}\left(q_{1}, q_{2}\right)-F^{j}\left(A_{n}, B_{n}\right)\right\|+\left\|F^{j}\left(A_{n}, B_{n}\right)-A_{n}\right\|+\left\|A_{n}-q_{1}\right\| \\
& \leq\left(k_{j}+1\right)\left[\left\|A_{n}-q_{1}\right\|+\left\|B_{n}-q_{2}\right\|\right]+\left\|F^{j}\left(A_{n}, B_{n}\right)-A_{n}\right\| \\
& \leq \frac{2}{n}\left(k_{j}+1\right)+\left\|F^{j}\left(A_{n}, B_{n}\right)-A_{n}\right\| .
\end{aligned}
$$

Taking the limit superior in the above inequality, from (2.6) we have

$$
\left\|F^{j}(q)-q_{1}\right\| \leq\left(k_{j} / 2\right) f^{-1}\left(2 r k_{j}\left(2-k_{j}^{-1}\right)\right) .
$$

Finally, the limit superior in the above inequality yields $\limsup _{j \rightarrow+\infty}\left\|F^{j}(q)-q_{1}\right\| \leq f^{-1}(0)=$ 0 , which implies that $\left\|F^{j}(q)-q_{1}\right\| \rightarrow 0$ as $j \rightarrow+\infty$.

Similar computations yield that $\left\|F^{j}(\bar{q})-q_{2}\right\| \rightarrow 0$ as $j \rightarrow+\infty$. Hence,

$$
\left\{\begin{array}{l}
F\left(q_{1 j}, q_{2 j}\right) \rightarrow q_{1}, \\
F\left(q_{2 j}, q_{1 j}\right) \rightarrow q_{2} .
\end{array}\right.
$$

Since $F$ is continuous, we have

$$
\left\{\begin{array}{l}
q_{1}=\lim _{j \rightarrow+\infty} F\left(q_{1_{j+1}}, q_{2 j+1}\right)=\lim _{j \rightarrow+\infty} F\left(F\left(q_{1 j}, q_{2 j}\right), F\left(q_{2 j}, q_{1 j}\right)\right)=F\left(q_{1}, q_{2}\right), \\
q_{2}=\lim _{j \rightarrow+\infty} F\left(q_{2_{j+1}}, q_{1_{j+1}}\right)=\lim _{j \rightarrow+\infty} F\left(F\left(q_{2 j}, q_{1 j}\right), F\left(q_{1 j}, q_{2 j}\right)\right)=F\left(q_{2}, q_{1}\right) .
\end{array}\right.
$$

Hence $q=\left(q_{1}, q_{2}\right)$ is a coupled fixed point of $F$, which completes the proof.

The conclusion of demi-closedness in the previous theorem states that if sequences $\left\{x_{n}\right\}$ and $\left\{y_{n}\right\}$ in $D$ are such that $x_{n} \rightarrow q_{1}, y_{n} \rightarrow q_{2}$ weakly and $x_{n}-F\left(x_{n}, y_{n}\right) \rightarrow 0, y_{n}-F\left(y_{n}, x_{n}\right) \rightarrow$ 0 strongly, then $\left(q_{1}, q_{2}\right)$ is a coupled fixed point of $F$. A more direct conclusion about the existence of a coupled fixed point of $F$ can be obtained by adding the property boundedness of the subset $D$. This fact is expressed in the following theorem. 
Theorem 2.2 Let D be a nonempty convex closed and bounded subset of a uniformly convex Banach space E. Then any asymptotically nonexpansive mapping $T: D \times D \rightarrow D$ has a coupled fixed point.

Proof Let $u, v \in D$ be fixed. Define the set $R(u, v)$ as follows:

$$
R(u, v)=\left\{\rho \in \mathbb{R} / \exists k_{\rho} \in \mathbb{N}: D \cap\left(\bigcap_{i=k_{\rho}}^{+\infty} S\left(F^{i}(u, v)+F^{i}(v, u), \rho\right)\right) \neq \emptyset\right\},
$$

where $S(x, r)$ is the open sphere in $E$ of center $x$ and radius $r$. $D$ is bounded, so if $d:=\operatorname{diam} D$ (diameter of $D), d \in R(u, v)$, hence $R(u, v) \neq \emptyset$. Let $\rho^{*}$ be the g.l.b. of $R(u, v)$. For each $\epsilon>0$, define the set

$$
A_{\epsilon}:=\bigcup_{k=1}^{+\infty}\left(\bigcap_{i=k}^{+\infty} S\left(F^{i}(u, v)+F^{i}(v, u), \rho^{*}+\epsilon\right)\right) .
$$

The sets $A_{\epsilon} \cap D$ are nonempty and convex. Since $E$ is reflexive, $A:=\bigcap_{\epsilon>0} \bar{A}_{\epsilon} \cap D$ is nonempty. Now, for any $x, y \in A$ and $\eta>0$, there exists $N \in \mathbb{N}$ such that $i \geq N$ implies that $\left\|x-F^{i}(u, v)\right\|+\left\|y-F^{i}(v, u)\right\| \leq \rho^{*}+\eta$.

Let us show that there exist $x, y \in A$ such that the sequences $\left\{F^{n}(x, y)\right\}$ and $\left\{F^{n}(y, x)\right\}$ converge to $x$ and $y$ respectively. Suppose that for all $x, y \in A$ such sequences do not converge. Then $\exists \epsilon>0$ and a strictly increasing sequence of integers $\left\{n_{i}\right\}$ is such that

$$
\left\|F^{n_{i}}(x, y)-x\right\|+\left\|F^{n_{i}}(y, x)-y\right\| \geq \epsilon, \quad i=1,2, \ldots
$$

For $m>n$,

$$
\begin{aligned}
\left\|F^{n}(x, y)-F^{m}(y, x)\right\| & =\left\|F^{n}(x, y)-F^{n}\left(F^{m-n}(x, y), F^{m-n}(y, x)\right)\right\| \\
& \leq k_{n} \frac{\left\|x-F^{m-n}(x, y)\right\|+\left\|y-F^{m-n}(y, x)\right\|}{2}, \\
\left\|F^{n}(y, x)-F^{m}(y, x)\right\| & =\left\|F^{n}(y, x)-F^{n}\left(F^{m-n}(y, x), F^{m-n}(y, x)\right)\right\| \\
& \leq k_{n} \frac{\left\|x-F^{m-n}(x, y)\right\|+\left\|y-F^{m-n}(y, x)\right\|}{2} .
\end{aligned}
$$

Hence

$$
\begin{aligned}
& \left\|F^{n}(y, x)-F^{m}(y, x)\right\|+\left\|F^{n}(y, x)-F^{m}(y, x)\right\| \\
& \quad \leq k_{n}\left[\left\|x-F^{m-n}(x, y)\right\|+\left\|y-F^{m-n}(y, x)\right\|\right] .
\end{aligned}
$$

Let $\delta$ be the modulus of convexity of the space $E$. Assume $\rho^{*}>0$ and $\alpha>0$ so that $(1-$ $\left.\delta\left(\frac{\epsilon}{\rho^{*}+\alpha}\right)\right)\left(\rho^{*}+\alpha\right)<\rho^{*}$, and select $n$ so that $\left\|x-F^{n}(x, y)\right\|+\left\|y-F^{n}(y, x)\right\| \geq \epsilon$ and so that $k_{n}\left(\rho^{*}+\frac{\alpha}{2}\right) \leq \rho^{*}+\alpha$.

If $N \geq n$ is sufficiently large, then $m>N$ implies that

$$
\left\|x-F^{m-n}(u, v)\right\|+\left\|y-F^{m-n}(v, u)\right\| \leq \rho^{*}+\frac{\alpha}{2},
$$


and also

$$
\begin{aligned}
\left\|F^{n}(x, y)-F^{m}(u, v)\right\|+\left\|F^{n}(y, x)-F^{m}(v, u)\right\| \leq & k_{n}\left[\left\|x-F^{m-n}(u, v)\right\|\right. \\
& \left.+\left\|y-F^{m-n}(v, u)\right\|\right] \\
\leq & k_{n}\left(\rho^{\prime \prime}+\frac{\alpha}{2}\right) \\
\leq & \rho^{*}+\alpha .
\end{aligned}
$$

Now, by the uniform convexity of $E$,

$$
\left\|\frac{X+Y}{2}\right\| \leq\left[1-\delta\left(\frac{\epsilon}{\rho^{*}+\alpha}\right)\right] \frac{\epsilon}{\rho^{*}+\alpha}, \quad \text { for }\|X\|,\|Y\| \leq \rho^{*}+\alpha,\|X-Y\| \geq \epsilon .
$$

Since $\left\|x-F^{m}(u, v)\right\|+\left\|y-F^{m}(v, u)\right\| \leq \rho^{*}+\frac{\alpha}{2}$, letting $X=x-F^{m}(u, v)+y-F^{m}(v, u)$ and $Y=F^{n}(x, y)+F^{n}(y, x)-F^{m}(u, v)-F^{m}(v, u)$, we have if $m>N$

$$
\left\|\frac{x+F^{n}(x, y)+y+F^{n}(y, x)}{2}-\left(F^{m}(u, v)+F^{m}(v, u)\right)\right\| \leq\left(1-\delta\left(\frac{\epsilon}{\rho^{*}+\alpha}\right)\right)\left(\rho^{\prime \prime}+\alpha\right)<\rho^{\prime \prime} .
$$

This contradicts the definition of $\rho^{\prime \prime}$, the g.l.b. of $R(u, v)$, since

$$
\rho^{*}>\left(1-\delta\left(\frac{\epsilon}{\rho^{*}+\alpha}\right)\right)\left(\rho^{*}+\alpha\right) \in R(u, v) \text {. }
$$

Hence, $\rho^{*}=0$ and so $x=F(x, y)$ and $y=F(x, y)$. The fact that $\rho^{*}=0$ implies that the sequences $\left\{F^{n}(u, v)\right\}$ and $\left\{F^{n}(v, u)\right\}$ are Cauchy, hence $F^{n}(u, v) \rightarrow x=F(x, y)$ and $F^{n}(v, u) \rightarrow$ $y=F(y, x)$. This completes the proof.

Since nonexpansive maps are asymptotically nonexpansive, we have the following corollary.

Corollary 2.3 Let D be a nonempty convex closed and bounded subset of a uniformly convex Banach space. Then any nonexpansive mapping $T: D \times D \rightarrow D$ has a coupled fixed point.

Remark 2.4 Theorem 2.1 is an extension of Theorem 1 of [5] to nonexpansive maps defined in a product space. The proof of Theorem 2.2 follows the methodology in [4], extending the result therein to product spaces. Our results are, to the best of our knowledge, first of their kind in the theory of nonexpansiveness in product spaces dealing with the existence of a coupled fixed point. 


\section{Author details}

${ }^{1}$ Department of Mathematics, University of Lagos, Akoka, Lagos, Nigeria. ${ }^{2}$ College of Statistics and Mathematics, Yunnan University of Finance and Economics, Kunming, Yunnan 650221, China.

Received: 21 September 2012 Accepted: 1 February 2013 Published: 25 March 2013

\section{References}

1. Bruck, RE: A simple proof of the mean ergodic theorem for nonlinear contractions in Banach spaces. Isr. J. Math. 32, 107-116 (1979)

2. Bruck, RE: On the convex approximation property and the asymptotic behavior of nonlinear contractions in Banach spaces. Isr. J. Math. 38(4), 304-314 (1981)

3. Zarantonello, EH: Protections on convex sets in Hilbert space and spectral theory. In: Contributions to Nonlinear Functional Analysis, pp. 237-244. Academic Press, New York (1971)

4. Goebel, K, Kirk, WA: A fixed point theorem for asymptotically nonexpansive mappings. Proc. Am. Math. Soc. 35, 171-174 (1972)

5. Chang, SS, Cho, YJ, Zhao, H: Demi-closed principle and weak convergence problems for asymptotically nonexpansive mappings. J. Korean Math. Soc. 38(6), 1245-1260 (2001)

6. Bhaskar, TG, Lakshmikantham, V: Fixed point theorems in partially ordered metric spaces and applications. Nonlinear Anal. 65, 1379-1393 (2006)

7. Chang, SS, Cho, YJ, Huang, NJ: Coupled fixed point theorems with applications. J. Korean Math. Soc. 33(3), 575-585 (1996)

8. Guo, D, Lakshmikantham, V: Coupled fixed points of nonlinear operators with applications. Nonlinear Anal. TMA 11 623-632 (1987)

9. Oka, $\mathrm{H}$ : An ergodic theorem for asymptotically nonexpansive mappings in the intermediate sense. Proc. Am. Math Soc. $125,1693-1703$ (1997)

10. Giesy, DP: On a convexity condition in normed linear spaces. Trans. Am. Math. Soc. 125, 114-146 (1966)

doi:10.1186/1687-1812-2013-68

Cite this article as: Olaoluwa et al.: Coupled fixed point theorems for asymptotically nonexpansive mappings. Fixed Point Theory and Applications 2013 2013:68

\section{Submit your manuscript to a SpringerOpen ${ }^{\circ}$ journal and benefit from:}

- Convenient online submission

- Rigorous peer review

Immediate publication on acceptance

- Open access: articles freely available online

- High visibility within the field

- Retaining the copyright to your article 